\title{
Hemocompatibility of Silicon-Based Substrates for Biomedical Implant Applications
}

\author{
Lalitha Muthusubramaniam, ${ }^{1,2}$ Rachel Lowe, ${ }^{1}$ William H. Fissell,${ }^{3,4}$ Lingyan Li, ${ }^{3}$ \\ Roger E. Marchant, ${ }^{5}$ Tejal A. Desai, ${ }^{1,2}$ and Shuvo Roy ${ }^{1,2}$ \\ ${ }^{1}$ Department of Bioengineering and Therapeutic Sciences, University of California, San Francisco, Box 2520, QB3 Second Floor \\ BH203, San Francisco, CA 94158-2330, USA; ${ }^{2}$ Joint Graduate Group in Bioengineering, University of California, \\ San Francisco-University of California, Berkeley, San Francisco, CA, USA; ${ }^{3}$ Department of Biomedical Engineering, Cleveland \\ Clinic, Cleveland, OH, USA; ${ }^{4}$ Department of Nephrology and Hypertension, Cleveland Clinic, Cleveland, OH, USA; and \\ ${ }^{5}$ Department of Biomedical Engineering, Case Western Reserve University, Cleveland, OH, USA
}

(Received 15 September 2010; accepted 18 January 2011; published online 2 February 2011)

Associate Editor Angelique Louie oversaw the review of this article.

\begin{abstract}
Silicon membranes with highly uniform nanopore sizes fabricated using microelectromechanical systems (MEMS) technology allow for the development of miniaturized implants such as those needed for renal replacement therapies. However, the blood compatibility of silicon has thus far been an unresolved issue in the use of these substrates in implantable biomedical devices. We report the results of hemocompatibility studies using bare silicon, polysilicon, and modified silicon substrates. The surface modifications tested have been shown to reduce protein and/ or platelet adhesion, thus potentially improving biocompatibility of silicon. Hemocompatibility was evaluated under four categories - coagulation (thrombin-antithrombin complex, TAT generation), complement activation (complement protein, C3a production), platelet activation (P-selectin, CD62P expression), and platelet adhesion. Our tests revealed that all silicon substrates display low coagulation and complement activation, comparable to that of Teflon and stainless steel, two materials commonly used in medical implants, and significantly lower than that of diethylaminoethyl (DEAE) cellulose, a polymer used in dialysis membranes. Unmodified silicon and polysilicon showed significant platelet attachment; however, the surface modifications on silicon reduced platelet adhesion and activation to levels comparable to that on Teflon. These results suggest that surface-modified silicon substrates are viable for the development of miniaturized renal replacement systems.
\end{abstract}

Keywords-Surface modification, Coagulation, Complement, Platelet adhesion, Activation.

Address correspondence to Shuvo Roy, Department of Bioengineering and Therapeutic Sciences, University of California, San Francisco, Box 2520, QB3 Second Floor BH203, San Francisco, CA 94158-2330, USA. Electronic mail: Shuvo.Roy@ucsf.edu

\section{INTRODUCTION}

Silicon-based materials are being used in the fabrication of a wide range of biomedical devices for diagnostic and therapeutic applications. These include devices for drug delivery, ${ }^{3}$ neural electrodes,${ }^{27}$ and implantable sensors, ${ }^{5}$ many of which are being considered for use within the body. One such promising technology is the development of miniaturized renal replacement devices using silicon nanoporous membranes. ${ }^{8}$ Such devices allow for extended dialysis each day leading to better outcomes and improved quality of life for patients with kidney failure. Current technologies make use of polydisperse polymer membranes which are limited by the need for high driving pressures for circulation and clearance. In contrast, silicon membranes with highly uniform pore sizes fabricated using microelectromechanical systems (MEMS) technology offer a promising approach for the production of miniaturized, high-performance renal replacement systems. ${ }^{19}$ Moreover, these membranes have been shown to support the growth and function of human renal proximal tubule cells indicating that they do not exhibit cytotoxicity. ${ }^{8}$

However, it is important that the single crystal silicon substrates display minimal adverse reactions in terms of blood compatibility (low surface coagulation, complement and/or platelet activation, and platelet adhesion) for effective use as the membrane material. Blood material interactions are especially important for hemodialysis due to the chronic exposure of blood to the membrane surface during treatment. ${ }^{4}$ Here we evaluate the blood compatibility of solid silicon 
substrates with and without surface modification by polymer conjugation. We have examined four types of surface modifications in this study, all of which have been shown to improve biocompatibility by preventing protein and/or platelet adhesion on the underlying surface. The first two are polyethylene glycol (PEG) conjugated to the surface using liquid ${ }^{23}$ and vaporbased deposition techniques, respectively. PEG has been shown to reduce non-specific protein adsorption and fouling on silicon surfaces. ${ }^{7}$ Solvent-free vapor deposition of PEG has been demonstrated to reduce protein adsorption by up to $80 \%$ on silicon-based surfaces. ${ }^{25}$ Furthermore, PEG films have shown longterm resistance to biofouling by reducing protein adsorption on silicon surfaces for up to four weeks under aqueous in vivo-like conditions. ${ }^{29}$

The third polymer is a glycocalyx-mimetic dextranmodified polyvinylamine (PVAm) surfactant which has been shown to reduce platelet adhesion. ${ }^{10} \mathrm{~A}$ zwitterionic polymer, polysulfobetaine methacrylate (pSBMA), shown to have good antifouling properties, was the fourth surface modification tested in the study. ${ }^{2}$ Additionally, polysilicon substrates used in MEMS fabrication to create uniformly porous membranes $^{7}$ were also included in this study.

Previous studies that have examined blood compatibility of silicon primarily focused on platelet adhesion tests of silicon-based substrates under static conditions. ${ }^{33,35}$ Here we extend these studies to investigate various aspects of hemocompatibility such as coagulation and complement activation, in addition to platelet activation. Accordingly, the surfaces were incubated with freshly drawn anticoagulated human whole blood or platelet-rich plasma (PRP) and tested for four different markers reflecting the following test categories - coagulation (thrombin-antithrombin complex, TAT generation), complement activation (formation of activated complement protein $\mathrm{C} 3 \mathrm{a}$ ), platelet activation (expression of P-selectin, CD62P), and platelet adhesion.

Hemocompatibility of the silicon-based substrates was compared to Teflon (polytetrafluoroethylene, PTFE) and medical grade stainless steel, two substrates that are routinely used in various medical implants. ${ }^{15,16}$ Both these surfaces display excellent blood compatibility ${ }^{32,34}$ and serve as negative controls in this study. Diethylaminoethyl (DEAE) cellulose, a polymer used in commercially available dialysis membranes ${ }^{4}$ was also tested to compare the performance of the silicon-based substrates with a conventional dialyzer material. Previously it has been shown that DEAE cellulose has advantages in terms of low platelet and complement activation, but exhibits high levels of TAT generation. ${ }^{4,28}$ Thus, it is not the ideal choice of dialyzer material with respect to surface coagulation.
Other polymer membranes such as polysulfone have more favorable coagulation properties in comparison to DEAE cellulose. ${ }^{4}$ However, this material was selected as the reference substrate because it could also serve as a positive control in our experiments. Adenosine diphosphate (ADP) is a known agonist of platelet activation. ${ }^{22}$ Upon activation, ADP is released from the dense granules of platelets, and aids in platelet aggregation and further activation. Hence, ADP was used as a positive control for platelet activation studies.

\section{MATERIALS AND METHODS}

\section{Materials}

All chemicals were purchased from Sigma-Aldrich (St. Louis, MO, USA) unless specified. Poly $(N$-vinyl dextran aldonamide- $c o-N$-vinyl hexanoamide) (PVAmDex/Hex) was purchased from Greatbatch, Inc. (Clarence, NY, USA) and 2-bromo-2-methyl- $N$-3[(trimethoxysilyl)propyl]-propanamide (BrTMOS) was synthesized according to literature. ${ }^{17}$ Virgin, primegrade, single side polished, $500 \mu \mathrm{m}$ thick, $\langle 100\rangle$-oriented, n-type, silicon wafers were used in the study. Deposition of polysilicon was performed at Cleveland Clinic. DEAE cellulose (Whatman, Piscataway, NJ, USA), Teflon (Scientific Commodities, Lake Havasu City, AZ, USA) and stainless steel (Feather Safety Razor, Medical Division, Kita-ku, Osaka, Japan) were used as reference materials.

\section{Substrate Preparation}

Liquid-based deposition of PEG on silicon was performed as described in previously published reports. ${ }^{23}$ Briefly, the single crystal silicon substrates were first sonicated in $70 \%$ ethanol for $10 \mathrm{~min}$ and then dried with nitrogen. Substrates were then oxidized in a $20: 80$ hydrogen peroxide $(30 \%)$ and sulfuric acid $(96 \%)$ mixture for $10 \mathrm{~min}$ at $120^{\circ} \mathrm{C}$. Substrates were then washed and sonicated for $10 \mathrm{~min}$ in deionized water and dried with nitrogen. Immediately, silicon substrates were immersed in a solution of $3 \mathrm{mM}$ 2-[methoxy(polyethyleneoxy)propyl]trimethoxysilane in toluene for $18 \mathrm{~h}$ at room temperature. Finally, the substrates were extensively washed with toluene, ethanol, and deionized water, before being dried with nitrogen to prepare the PEG-conjugated substrates.

Vapor-based deposition of PEG on silicon was performed at UCSF according to protocols developed in our laboratory. Single crystal silicon substrates were cleaned with a $3: 1$ sulfuric acid:hydrogen peroxide solution, rinsed in deionized water, and dried with 
nitrogen. Silicon pieces were then treated with oxygen plasma ( $200 \mathrm{~W}, 0.5 \mathrm{mTorr}$ ) for $5 \mathrm{~min}$ prior to being placed in the Teflon deposition chamber. The deposition chamber was purged with nitrogen, and a hot plate was used to maintain silicon surface temperature of $120^{\circ} \mathrm{C}$. [2-Methoxy(polyethyleneoxy)propyl] trimethoxysilane (PEG-silane) was vaporized at $250{ }^{\circ} \mathrm{C}$ in a closed stainless steel loop and subsequently released into the deposition chamber. Four vapor exposures for $30 \mathrm{~min}$ each were performed before silicon samples were removed for use.

PVAm-modified silicon substrates were prepared at Cleveland Clinic according to protocols published in our laboratory. ${ }^{18}$ Briefly, single crystal silicon wafers were plasma cleaned and placed in a solution of octadecyltrichlorosilane for $30 \mathrm{~min}$. The wafers were then washed twice with dried chloroform before immersion into an aqueous solution of PVAm-Dex/ Hex for $24 \mathrm{~h}$. The substrates were finally rinsed with deionized water and air dried to prepare the PVAmmodified silicon substrates.

The pSBMA-modified substrates were also fabricated at Cleveland Clinic using protocols published by our laboratory. ${ }^{17}$ Cleaned wafers of single crystal silicon were placed in an anhydrous bicyclohexyl solution of BrTMOS for $2 \mathrm{~h}$, rinsed, air dried and then placed in a flask under nitrogen. Polymerization was carried out using a solution of [2-(methacryloyloxy)ethyl]dimethyl(3-sulfopropyl)ammonium hydroxide monomer (SBMA) and 2,2'-bipyridyl (BPY) dissolved in a degassed solution of methanol and deionized water, to which copper(II) bromide and copper(I) bromide had been added. The substrates were then rinsed with ethanol and water and stored in deionized water overnight and then air dried for use in the study.

The surface of a silicon wafer oxidizes to form silicon dioxide when exposed to air. Our unmodified silicon surfaces are hence $\mathrm{Si} / \mathrm{SiO}_{2}$ surfaces. The unmodified silicon substrates were cleaned with piranha $\left(3: 1 \mathrm{H}_{2} \mathrm{SO}_{4} / \mathrm{H}_{2} \mathrm{O}_{2}\right.$ mixture) and dried with nitrogen before use. All substrates (except DEAE cellulose) were rinsed with $70 \%$ ethanol and dried before use. The substrates were all sterilized using UV radiation for 5 min prior to blood or plasma incubation.

\section{Blood Collection}

Blood was drawn at the UCSF Blood Center from healthy human donors using 3.8\% citrate (Fisher Scientific, Pittsburgh, PA, USA) or $17 \mathrm{IU} / \mathrm{mL}$ heparin (Fisher Scientific, Pittsburgh, PA, USA) as anticoagulant. Informed consent was obtained from donors prior to blood donation. Heparin is a commonly used anticoagulant in blood compatibility testing of biomaterials in vitro ${ }^{9,31}$ and was selected as the anticoagulant for
TAT generation studies. Unfractionated heparin (UFH) binds antithrombin and converts it into a more efficient inhibitor of thrombin and other coagulation factors such as factor Xa, factor IXa, and factor XIIa. ${ }^{11}$ At high concentrations, heparin binds heparin cofactor II to inhibit thrombin independent of antithrombin; however, it also binds to platelets and induces their aggregation. Heparin also binds anaphylotoxins such as the complement protein $\mathrm{C} 3 \mathrm{a} .{ }^{30}$ Sodium citrate neutralizes coagulation factor activation. Since citrate is a calcium chelator, it reduces the ionized calcium concentration in plasma leading to enhanced GPIIb/IIIa binding and inhibition of platelet aggregation. ${ }^{24}$ Thus, citrate does not cause spontaneous platelet activation in vitro and any activation that is seen is due to the surface of the biomaterial itself. For these reasons, citrate was selected for the platelet and complement activation studies.

Blood samples were stored on ice until the start of experiments based on previously published reports in literature, ${ }^{1,20}$ and in accordance with the guidelines of the International Standard ISO 10993 Part 4-Selection of tests for interactions with blood. ${ }^{13}$ All blood samples were handled in a similar manner and stored on ice for an equivalent amount of time $(60 \pm 5 \mathrm{~min})$. Whole blood was centrifuged at $1000 \mathrm{rpm}$ for $10 \mathrm{~min}$ at room temperature to obtain PRP for platelet adhesion studies. Platelet counts were obtained using a Hemavet950 (Drew Scientific, Oxford, CT, USA).

\section{Blood Incubation and Analysis}

Flow is the natural state of blood and flow studies are the ideal representation of conditions in vivo. Our experiments conducted under non-perfusion conditions are preliminary studies indicative of data obtained with flow-based studies. In flow-based systems, blood is exposed to additional foreign material such as tubing, and shear stress due to pumping, all of which have been shown to increase the activation of blood components. ${ }^{32}$ Streller et al. have shown that data obtained under non-flow conditions are representative of flow-based studies using control substrates such as Teflon, glass, and polyethersulfone. Considering these aspects, we decided to conduct preliminary studies under static conditions to examine the relative difference in activation levels between bare silicon and surface-modified silicon substrates. The substrates were, however, incubated on a gentle shaker (50 shakes per min) to avoid sedimentation of platelets. ${ }^{9,31}$

$400 \mu \mathrm{L}$ of whole blood from three donors was dispensed onto the substrates $(10 \mathrm{~mm} \times 10 \mathrm{~mm})$ placed in 24-well tissue culture polystyrene (TCPS) microplates. Four replicates were used for each donor for all substrates except polysilicon, where only three 
replicates were available for one of the donors due to inadvertent error in sample handling. For the C3a studies, substrates were mounted on Teflon spacers placed on tissue culture dishes to minimize possible activation from TCPS (Supplementary Figure S1). The substrates were incubated for $2 \mathrm{~h}$ at $37^{\circ} \mathrm{C}$ on a gentle shaker. Commercial ELISAs were used to characterize TAT generation (Enzygnost TAT micro, Siemens Healthcare Diagnostics, Deerfield, IL, USA) and C3a formation (Human C3a ELISA Kit, BD Biosciences, San Jose, CA, USA). Blood samples were mixed with specific inhibitors and centrifuged to collect plasma according to the manufacturer's protocols. Plasma samples were frozen at $-70{ }^{\circ} \mathrm{C}$ until further analysis.

\section{Surface Analysis after Incubation}

Platelet adhesion and activation was visualized using immunofluorescence staining for the platelet marker, CD41 (Abcam, Cambridge, MA, USA) and activated platelet marker, CD62P (Abcam, Cambridge, MA, USA). $200 \mu \mathrm{L}$ of PRP $\left(3.3 \times 10^{5}\right.$ platelets $\left./ \mu \mathrm{L}\right)$ from one donor was dispensed onto the substrates $(10 \mathrm{~mm} \times 10 \mathrm{~mm})$ mounted on Teflon spacers and incubated for $2 \mathrm{~h}$ at $37^{\circ} \mathrm{C}$ on a gentle shaker. Three replicates were used for all substrates except polysilicon, where only two replicates were available due to inadvertent error in sample handling. The platelets were treated with ADP in solution. ADP (40 $\mu \mathrm{M}$ final concentration in PRP) was added to PRP and dispensed on single crystal silicon $(10 \mathrm{~mm} \times 10 \mathrm{~mm})$ in triplicate for use as the positive control. After $2 \mathrm{~h}, \mathrm{PRP}$ was removed and the substrates were transferred to a 24-well plate and washed with phosphate-buffered saline (PBS). The platelets were fixed in 4\% paraformaldehyde (Fisher Scientific, Pittsburgh, PA, USA) for $15 \mathrm{~min}$, and blocked in $1 \%$ bovine serum albumin for $30 \mathrm{~min}$. Platelets were double labeled as follows: substrates were first incubated with antihuman CD62P mouse monoclonal antibody, diluted 1:50 in PBS for 60 min followed by Alexa Fluor 546 donkey anti-mouse antibody (Invitrogen, Carlsbad, CA, USA) diluted 1:100 in PBS for $60 \mathrm{~min}$. Finally the samples were incubated with antihuman CD41 FITC-labeled mouse monoclonal antibody diluted 1:300 in PBS for 60 min. Four images were acquired per replicate using a NikonEclipse Ti-E motorized inverted microscope to obtain a total of 12 images per substrate. Data analysis was performed using Fiji, an Open Source image analysis package based on ImageJ (http://pacific.mpi-cbg.de/).

\section{Statistical Analysis}

Statistically significant differences were estimated using analysis of variance (ANOVA) tests. For group differences shown to be significant by ANOVA, sequential Holm $t$-tests were performed to detect differences between specific pairs of substrates.

\section{RESULTS AND DISCUSSION}

\section{Surface Coagulation}

Activated thrombin is inhibited by antithrombin-III to form a proteinase/inhibitor complex (TAT), the concentration of which is used as a measure of thrombin activation and coagulation. ${ }^{32}$ Generation of TAT complex after whole blood incubation with the substrates was therefore determined to evaluate surface coagulation. As seen from Fig. 1, the most striking difference is that TAT generation is drastically reduced $(\sim 25$ - to 50 -fold, $p<0.001)$ on the silicon-based substrates when compared to DEAE cellulose. This indicates that the silicon-based substrates display superior anticoagulation properties when compared to the highly thrombogenic DEAE cellulose surface. Previously it has been shown that DEAE cellulose exhibits high levels of TAT generation. ${ }^{4,28}$ This is in good agreement with our data where DEAE cellulose acts as a positive control exhibiting high levels of TAT formation.

Most importantly, there is no significant difference in TAT generation with the single crystal silicon, modified silicon and polysilicon substrates when compared to Teflon and stainless steel. Thus, the siliconbased substrates display excellent anticoagulation, comparable to the negative control substrates.

\section{Complement Activation}

C3a anaphylatoxin is produced upon activation of the classical or alternate complement pathways. The amount of $\mathrm{C} 3 \mathrm{a}$ formed after whole blood incubation with the substrates is therefore used as a measure of complement activation. ${ }^{12}$ As seen from Fig. 2, C3a formation on the silicon-based substrates is substantially reduced $(\sim 3-$ to 4 -fold, $p<0.001)$ in comparison to DEAE cellulose. Previous studies have shown that modification of cellulose by the incorporation of DEAE groups offers certain advantages such as reduced complement activation. ${ }^{4,28}$ However, the silicon substrates exhibit even lower levels of C3a formation, indicating that they compare even more favorably than DEAE cellulose in terms of complement activation.

More importantly, there is no significant difference in C3a formation between the single crystal silicon, polysilicon and modified silicon substrates with respect to Teflon and stainless steel indicating that these substrates do not exhibit significant complement activation. 


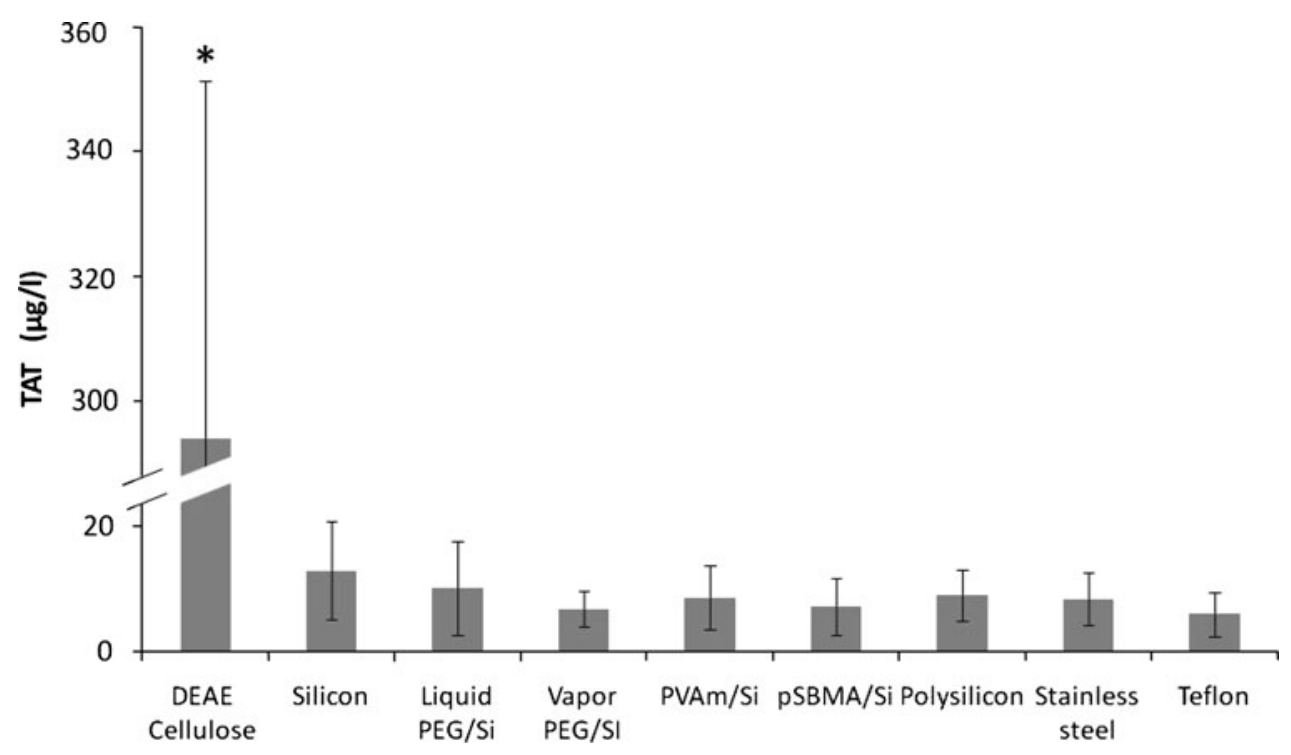

FIGURE 1. Surface coagulation presented as a measure of TAT generation after whole blood incubation with the substrates. Data is presented as average \pm standard deviation. ${ }^{*} p<0.001$ vs. Teflon.

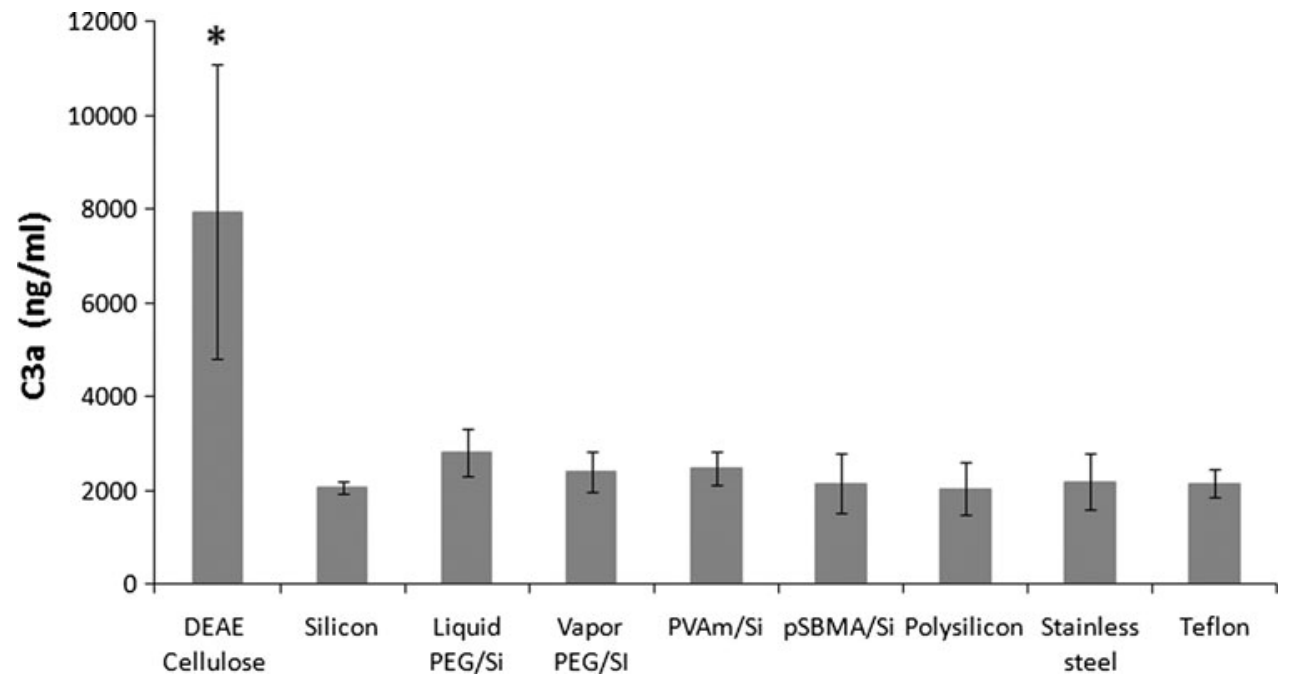

FIGURE 2. Complement activation presented as amount of C3a formed after whole blood incubation with the substrates. Data is presented as average \pm standard deviation. ${ }^{*} p<0.001$ vs. Teflon.

\section{Platelet Adhesion and Activation}

CD41 is an integrin heterodimer consisting of a heavy chain (GPIIb alpha) and a light chain (GPIIb beta) that is expressed on the surface of platelets. The alpha IIb chain forms a platelet glycoprotein complex with another molecule, CD61, and thus aids in platelet adhesion and aggregation. The CD62P antigen is a member of the selectin family of cellular adhesion molecules located in the storage granules of platelets. Upon activation, CD62P is released and stably expressed on the surface of activated platelets. Thus, CD41 and CD62P can be used as markers for platelet adhesion and activation, respectively. ${ }^{6}$ The fibrous texture of DEAE cellulose made it difficult to visualize platelet staining on this surface. Hence ADP, a known platelet activator, was used as the positive control for this study. Previous studies have established Teflon as a material that displays minimal platelet activation and thrombosis. ${ }^{32}$ Hence Teflon was selected as the as the negative control for further studies with platelet activation.

In this work, blood samples were stored on ice based on protocols from previously published reports in literature. ${ }^{1,20}$ Blood storage on ice is also in accordance with the guidelines of the International Standard ISO 10993 Part 4-Selection of tests for interactions 
with blood. ${ }^{13}$ Bergseth et al., report that the ideal conditions for storing blood samples collected for platelet activation studies is to place them on ice for 30 min. ${ }^{1}$ Another study that specifically looked at optimizing blood storage conditions reported that the percentage of $\mathrm{CD} 62 \mathrm{P}$ positive platelets does not change in anticoagulated blood samples stored at $4{ }^{\circ} \mathrm{C}$ over a period of $180 \mathrm{~min},{ }^{20}$ indicating that chilling does not change P-selectin levels at least over the time period relevant to our studies. In contrast, room temperature storage leads to the secretion of metabolic products such as lactate resulting in low $\mathrm{pH}$ and decreased platelet viability. ${ }^{14,26}$ At room temperature, platelets get activated as indicated by the secretion of beta-thromboglobulin, platelet factor-4, and P-selectin, all of which are known markers for platelet activation. For these aforementioned reasons, the choice was made to store the blood samples on ice until the start of experiments. Additionally, we observed minimal to no platelet activation on the surface-modified silicon substrates and Teflon control (Figs. $4 \mathrm{~d}-4 \mathrm{~h}$ ). Thus, it is unlikely that platelet activation is caused by storage of blood samples on ice.

As seen in Fig. 3, single crystal silicon and polysilicon display extensive platelet adhesion and activation as shown by labeling for CD41 (green), CD62P (red) and colocalization (Figs. 4a, 4b). However, platelet spreading on these two substrates is still considerably lesser compared to the ADP/silicon substrate (Fig. 4c). Also, the ratio of activated platelets on $\mathrm{ADP} /$ silicon $(0.92)$ is higher than that on either bare silicon $(0.8)$ or polysilicon (0.5), respectively (Fig. 5). Increase in platelet spreading has been correlated with higher levels of platelet activation. ${ }^{33}$ Thus, the dramatic increase in platelet size $(\sim 10$-fold as seen in Fig. 5, $p<0.001)$ on $\mathrm{ADP} /$ silicon compared to bare silicon or polysilicon establishes ADP as a good positive control for this study. Platelet spreading on the ADP/silicon surface also contributes to the lower platelet count per unit area as seen in Fig. 5.

Notably, the polymer-conjugated silicon substrates show minimal to no platelet coverage compared to the
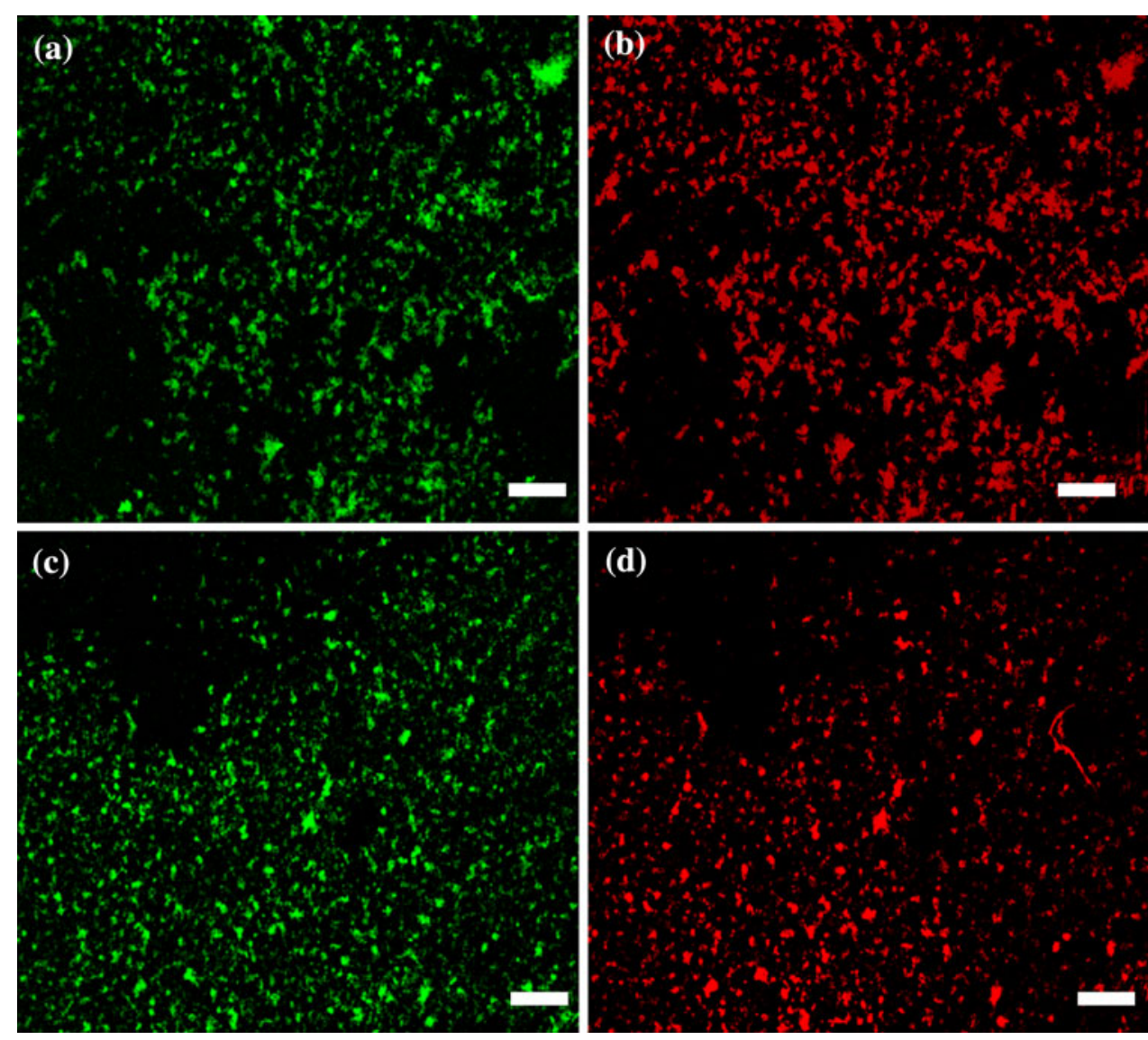

FIGURE 3. Representative images showing platelet adhesion and activation after incubation with PRP as visualized by immunofluorescence staining for CD41 (in green, platelet marker) and CD62P (in red, activated platelet marker). (a) Bare silicon substrate showing the FITC-labeled CD41 channel alone, (b) bare silicon substrate showing the Alexa 547-labeled CD62P channel alone, (c) and (d) are corresponding images in bare polysilicon substrate. Scale bars represent $10 \mu \mathrm{m}$. 

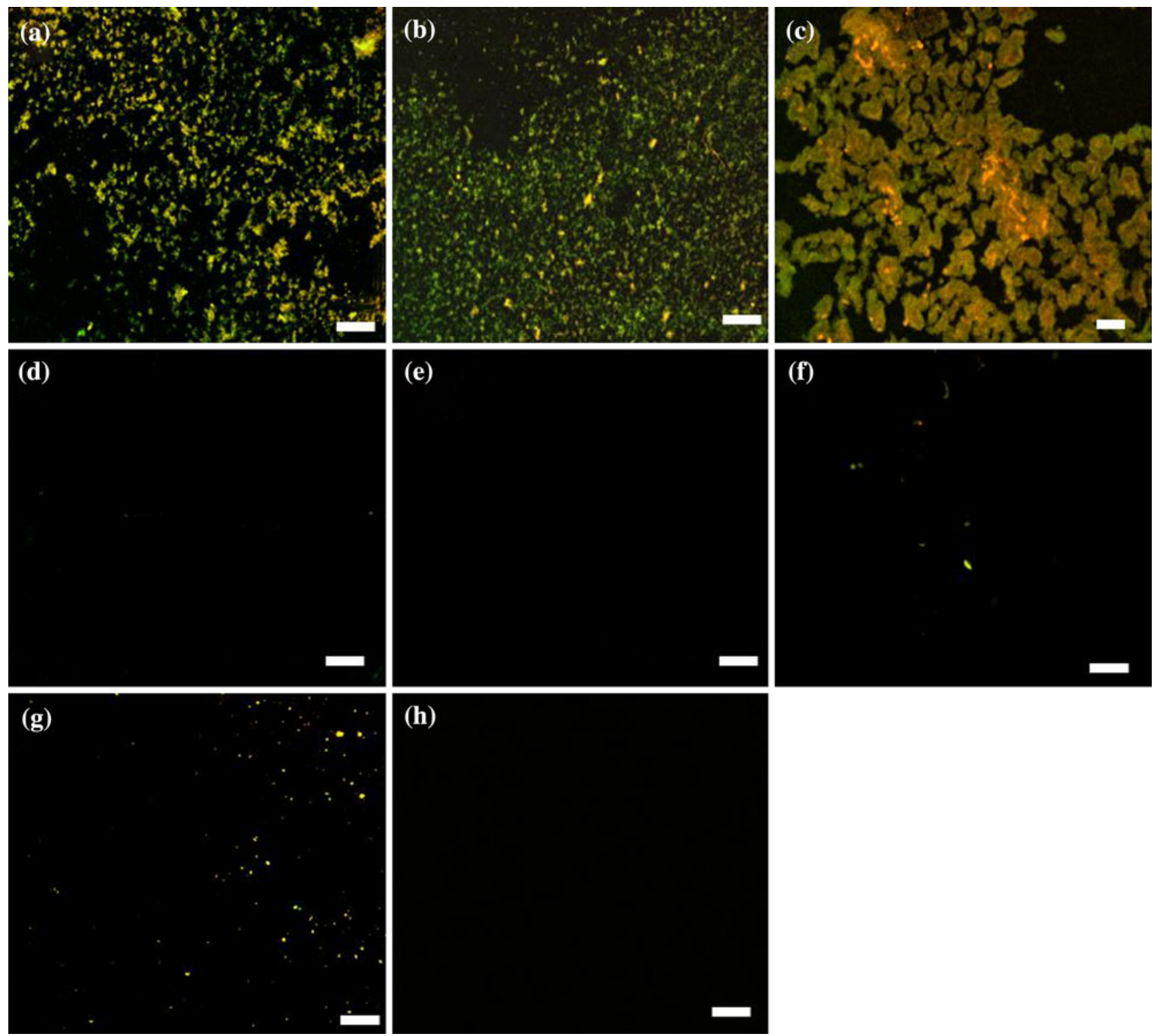

FIGURE 4. Superimposed images of the FITC-labeled CD41 (green) and Alexa 547-labeled CD62P (red) show colocalization of platelet adhesion and activation as seen in yellow: (a) Bare silicon, (b) bare polysilicon, (c) ADP/silicon, (d) liquid PEG/silicon, (e) vapor PEG/silicon, (f) PVAm/silicon, (g) pSBMA/silicon, and (h) Teflon. Scale bars represent $10 \mu \mathrm{m}$.

unmodified single crystal silicon substrate (Figs. 4d$4 \mathrm{~g}$ ). Platelet counts (both activated and non-activated) are also significantly lower on these surfaces compared to unmodified silicon as seen in Fig. $5(p<0.001)$. This indicates that surface modification significantly reduces platelet adhesion and activation on the silicon surface. Of the four types of modifications that were tested, pSBMA alone shows slightly higher levels of platelet adhesion and activation as seen from the platelet counts and the mean platelet size in Fig. 5. However, platelet counts on the pSBMA substrate are still 8-fold lower compared to that of unmodified silicon substrate.
Previously it has been shown that PEG modification significantly reduces protein adsorption and thrombosis on silicon-based substrates. ${ }^{7,25}$ Moreover, PEG films have been shown to retain their ability to reduce protein fouling for up to four weeks under aqueous in vivo-like conditions. ${ }^{29}$ Platelet adhesion on PVAm coated polycarbonate disks has been shown to be $\sim 90 \%$ less than that of uncoated disks. ${ }^{10}$ pSBMA grafted surfaces have also been shown to reduce platelet adhesion and activation. ${ }^{2}$ These results are in good agreement with our data indicating that surface modification can be used to significantly improve the hemocompatibility of silicon for implant devices. 


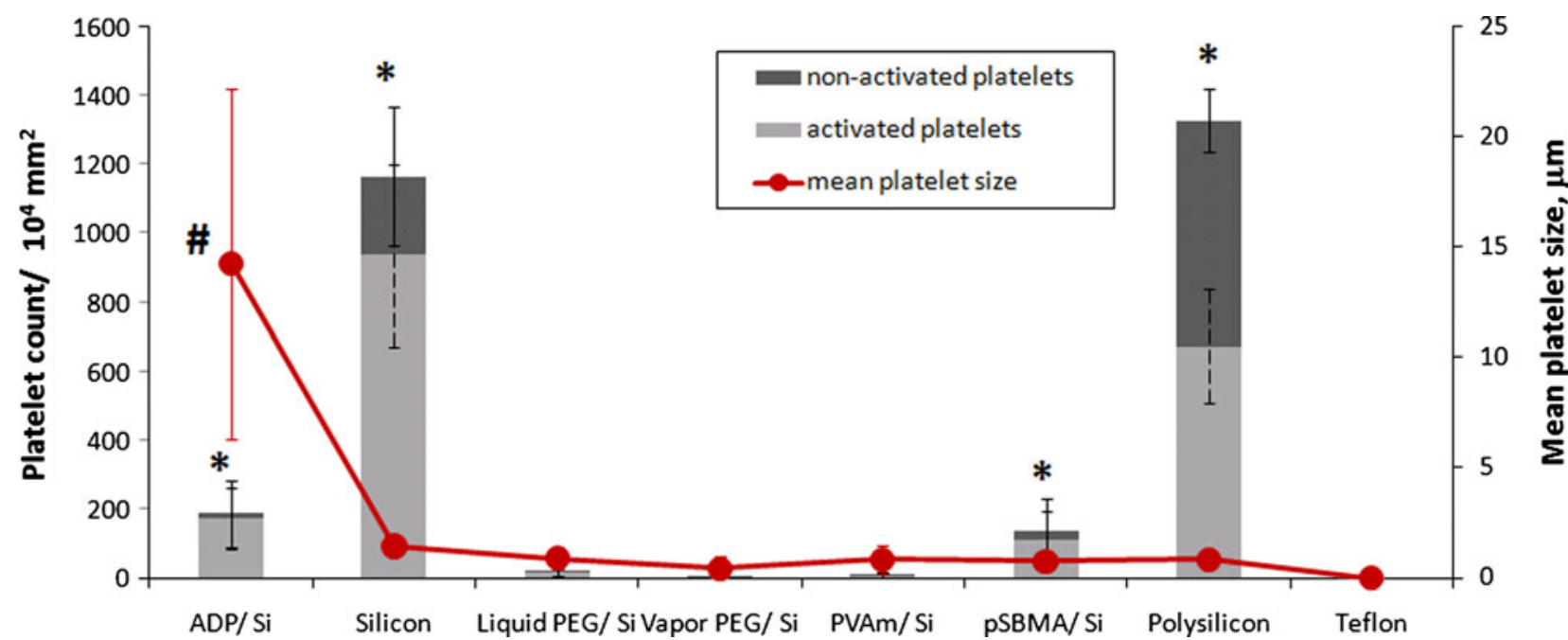

FIGURE 5. Platelet adhesion and activation after incubation of substrates with PRP: The vertical axis on the left represents the number of activated and non-activated platelets attached to the substrate as visualized by immunofluorescence staining for CD41 (platelet marker) and CD62P (activated platelet marker). Data is presented as average \pm standard deviation of 12 images. ${ }^{*} p<0.001$ vs. corresponding platelet counts in vapor PEG/silicon. The vertical axis on the right represents mean platelet size. Data is presented as average $\pm 95 \%$ confidence interval of 12 images. ${ }^{\#} p<0.001$ vs. corresponding size in vapor PEG/silicon. Teflon could not be used as reference because platelet count on Teflon was zero.

Of considerable interest is the fact that platelet adhesion and activation levels on the modified silicon substrates (with the exception of pSBMA) are not significantly different from that of Teflon (Fig. 4h). This result is encouraging as it indicates that surface modification reduces platelet activation on silicon to levels comparable to that of Teflon, a material that is extensively used in medical implant devices.

Pilot studies conducted in rats using silicon implants with and without PEG conjugation have shown that PEG-conjugated silicon implants showed no thrombus formation compared to bare silicon implants which had significant adherent thrombi. ${ }^{21}$ This data is in excellent agreement with our studies showing that surface modification using polymers such as PEG significantly reduces platelet adhesion and activation on silicon. While these results are very encouraging, these preliminary studies need to be extended to examine long-term blood compatibility of silicon membranes under flow-based conditions that are relevant to the end application of dialysis. Future work should also focus on investigating different markers of hemocompatibility in vivo to evaluate the feasibility of silicon membranes for use in implantable renal replacement systems.

\section{CONCLUSION}

Any device that is brought into contact with blood causes adverse reactions thus compromising the hemocompatibility of the device. Such reactions are particularly challenging in the case of hemodialyzers which come into chronic contact with blood. It is therefore very important to evaluate the blood compatibility of silicon surfaces before they can be used in the development of implantable renal replacement units. Our studies show that unmodified single crystal silicon and polysilicon substrates display low levels of coagulation and complement activation, comparable to that of Teflon and stainless steel-two materials extensively used in implant applications. Both these surfaces also perform considerably better in these aspects when compared to DEAE cellulose, a commercially available material used in dialysis membranes. The unmodified silicon substrates, however, display significantly higher levels of platelet activation compared to Teflon, although these values are still substantially lower than that with ADP ( 10 -fold), a known agonist of platelet activation.

Of considerable interest is the fact that silicon substrates modified with PEG and PVAm polymers showed excellent performance comparable to Teflon in all four aspects of hemocompatibility - surface coagulation, complement and platelet activation, and adhesion, respectively. Thus, surface modification improves the blood compatibility of silicon to levels comparable to medical grade implant materials such as Teflon. All the surface modifications that were tested (PEG, PVAm, and pSBMA) were also far superior to DEAE cellulose in terms of coagulation and complement activation. This is encouraging as it suggests that surface-modified silicon substrates have the potential to perform significantly better than some of the currently 
available materials used in dialyzers. Collectively, our results demonstrate that the surface-modified silicon substrates may be used in the development of membranes for implantable biomedical devices.

\section{ELECTRONIC SUPPLEMENTARY MATERIAL}

The online version of this article (doi:10.1007/ s10439-011-0256-y) contains supplementary material, which is available to authorized users.

\section{ACKNOWLEDGMENTS}

Funding for this work was provided by the NIH Grant R01EB008049. Partial support was provided by the Juvenile Diabetes Research Foundation (JDRF). The vapor deposited PEG-modified substrates were fabricated by D. Bernards (UCSF). We would like to thank J. Katz and her staff at the UCSF Blood Center for assistance with blood collection, D. Bernards and L. Walsh for their blood donation, and K. Thorn and A. Thwin at the UCSF Nikon Imaging Center for help with acquiring the platelet immunofluorescence data. We are also grateful to Dr. Kandice-Kottke Marchant of the Cleveland Clinic for helpful discussions and feedback.

\section{OPEN ACCESS}

This article is distributed under the terms of the Creative Commons Attribution Noncommercial License which permits any noncommercial use, distribution, and reproduction in any medium, provided the original author(s) and source are credited.

\section{REFERENCES}

${ }^{1}$ Bergseth, G., K. Lappegård, V. Videm, and T. E. Mollnes. A novel enzyme immunoassay for plasma thrombospondin: comparison with beta-thromboglobulin as platelet activation marker in vitro and in vivo. Thromb. Res. 99:4150,2000 .

${ }^{2}$ Chang, Y., S.-C. Liao, A. Higuchi, R.-C. Ruaan, C.-W. Chu, and W.-Y. Chen. A highly stable nonbiofouling surface with well-packed grafted zwitterionic polysulfobetaine for plasma protein repulsion. Langmuir 24:54535458, 2008.

${ }^{3}$ Desai, T. A., D. Hansford, and M. Ferrari. Characterization of micromachined silicon membranes for immunoisolation and bioseparation applications. J. Membr. Sci. 159:221-231, 1999.

${ }^{4}$ Erlenkötter, A., P. Endres, B. Nederlof, C. Hornig, and J. Vienken. Score model for the evaluation of dialysis membrane hemocompatibility. Artif. Organs 32:962-969, 2008.
${ }^{5}$ Ferrara, L. A., A. J. Fleischman, D. Togawa, T. W. Bauer, E. C. Benzel, and S. Roy. An in vivo biocompatibility assessment of MEMS materials for spinal fusion monitoring. Biomed. Microdevices 5:297-302, 2003.

${ }^{6}$ Ferraz, N., M. K. Ott, and J. Hong. Time sequence of blood activation by nanoporous alumina: studies on platelets and complement system. Microsc. Res. Tech. 73:1101-1109, 2010.

${ }^{7}$ Fissell, W. H., A. Dubnisheva, A. N. Eldridge, A. J. Fleischman, A. L. Zydney, and S. Roy. High-performance silicon nanopore hemofiltration membranes. J. Membr. Sci. 326:58-63, 2009.

${ }^{8}$ Fissell, W. H., A. J. Fleischman, H. D. Humes, and S. Roy. Development of continuous implantable renal replacement: past and future. Transl. Res. 150:327-336, 2007.

${ }^{9}$ Gouzy, M. F., C. Sperling, K. Salchert, T. Pompe, U. Streller, P. Uhlmann, C. Rauwolf, F. Simon, F. Böhme, B. Voit, and C. Werner. In vitro blood compatibility of polymeric biomaterials through covalent immobilization of an amidine derivative. Biomaterials 25:3493-3501, 2004.

${ }^{10}$ Gupta, A. S., S. Wang, E. Link, E. H. Anderson, C. Hofmann, J. Lewandowski, K. Kottke-Marchant, and R. E. Marchant. Glycocalyx-mimetic dextran-modified poly(vinyl amine) surfactant coating reduces platelet adhesion on medical-grade polycarbonate surface. Biomaterials 27:3084-3095, 2006.

${ }^{11}$ Hirsh, J., R. Raschke, T. E. Warkentin, J. E. Dalen, D. Deykin, and L. Poller. Heparin: mechanism of action, pharmacokinetics, dosing considerations, monitoring, efficacy, and safety. Chest 108:258S-275S, 1995.

${ }^{12}$ Hong, J., K. Nilsson Ekdahl, H. Reynolds, R. Larsson, and B. Nilsson. A new in vitro model to study interaction between whole blood and biomaterials. Studies of platelet and coagulation activation and the effect of aspirin. Biomaterials 20:603-611, 1999.

${ }^{13}$ ISO Biological evaluation of medical devices: Part 4 Selection of tests for interactions with the blood. 2002.

${ }^{14}$ Josefsson, E. C., J. H. Hartwig, and K. M. Hoffmeister. Platelet storage temperature-how low can we go? Transfus. Med. Hemother. 34:253-261, 2007.

${ }^{15}$ Jung, Y. G., H. Y. Kim, H. J. Dhong, K. N. Park, H. J. Lee, Y. J. Lim, and J. Y. Min. Ultrasonographic monitoring of implant thickness after augmentation rhinoplasty with expanded polytetrafluoroethylene. Am. J. Rhinol. Allergy 23:105-110, 2009.

${ }^{16}$ Kenny, D., M. C. Hamilton, and R. P. Martin. Stainless steel stents and magnetic resonance imaging looking into a black hole. J. Am. Coll. Cardiol. 54:2202-2203, 2009.

${ }^{17}$ Li, L., R. E. Marchant, A. Dubnisheva, S. Roy, and W. H. Fissell. Anti-biofouling sulfobetaine polymer thin films on silicon and silicon nanopore membranes. J. Biomater. Sci. Polym. Ed. 22:91-106, 2010.

${ }^{18}$ Li, L., J. Zhu, R. E. Marchant, S. Roy, and W. H. Fissell. Biomimetic non-adhesive oligosaccharide surfactant polymer on silicon nanopore membranes for hemofiltration. J. Biomed. Mater. Res., Part A, 2010 (in press).

${ }^{19}$ Lopez, C. A., A. J. Fleischman, S. Roy, and T. A. Desai. Evaluation of silicon nanoporous membranes and ECMbased microenvironments on neurosecretory cells. Biomaterials 27:3075-3083, 2006.

${ }^{20}$ Macey, M., U. Azam, D. McCarthy, L. Webb, E. S. Chapman, D. Okrongly, D. Zelmanovic, and A. Newland. Evaluation of the anticoagulants EDTA and citrate, theophylline, adenosine, and dipyridamole (CTAD) for 
assessing platelet activation on the ADVIA 120 hematology system. Clin. Chem. 48:891-899, 2002.

${ }^{21}$ Melvin, M. E., W. H. Fissell, S. Roy, and D. L. Brown. Silicon induces minimal thromboinflammatory response during 28-day intravascular implant testing. ASAIO J. 56:344-348, 2010.

${ }^{22}$ Nilsson, P. H., A. E. Engberg, J. Bäck, L. Faxälv, T. L. Lindahl, B. Nilsson, and K. N. Ekdahl. The creation of an antithrombotic surface by apyrase immobilization. Biomaterials 31:4484-4491, 2010.

${ }^{23}$ Papra, A., N. Gadegaard, and N. B. Larsen. Characterization of ultrathin poly(ethylene glycol) monolayers on silicon substrates. Langmuir 17:1457-1460, 2001.

${ }^{24}$ Phillips, D. R., W. Teng, A. Arfsten, L. Nannizzi-Alaimo, M. M. White, C. Longhurst, S. J. Shattil, A. Randolph, J. A. Jakubowski, L. K. Jennings, and R. M. Scarborough. Effect of $\mathrm{Ca}^{2+}$ on GP IIb-IIIa interactions with integrilin: enhanced GP IIb-IIIa binding and inhibition of platelet aggregation by reductions in the concentration of ionized calcium in plasma anticoagulated with citrate. Circulation 96:1488-1494, 1997.

${ }^{25}$ Popat, K. C., and T. A. Desai. Poly(ethylene glycol) interfaces: an approach for enhanced performance of microfluidic systems. Biosens. Bioelectron. 19:1037-1044, 2004.

${ }^{26}$ Rumjantseva, V., and K. M. Hoffmeister. Novel and unexpected clearance mechanisms for cold platelets. Transfus. Apher. Sci. 42:63-70, 2010.

${ }^{27}$ Santiesteban, F. M. M., S. D. Swanson, D. C. Noll, and D. J. Anderson. Magnetic resonance compatibility of multichannel silicon microelectrode systems for neural recording and stimulation: design criteria, tests, and recommendations. IEEE Trans. Biomed. Eng. 53:547-558, 2006.
${ }^{28}$ Schultze, G., S. Hollmann, and P. Sinah. Formation of thrombin-antithrombin III complex using polyamide and hemophan dialyzers. Int. J. Artif. Organs 15:370-373, 1992.

${ }^{29}$ Sharma, S., R. W. Johnson, and T. A. Desai. Evaluation of the stability of nonfouling ultrathin poly(ethylene glycol) films for silicon-based microdevices. Langmuir 20:348-356, 2003.

${ }^{30}$ Sinitsyn, V. V., E. T. Bokchubaev, A. G. Mamontova, N. V. Ovtrakht, E. L. Nasonov, G. A. Konovalov, and V. V. Kukharchuk. C3a and C5a anaphylatoxins bind to heparinbased sorbent in low density lipoprotein apheresis: in vitro and in vivo investigations. Artif. Organs 16:291-293, 1992.

${ }^{31}$ Sperling, C., M. Fischer, M. F. Maitz, and C. Werner. Blood coagulation on biomaterials requires the combination of distinct activation processes. Biomaterials 30:44474456, 2009.

${ }^{32}$ Streller, U., C. Sperling, J. Hübner, R. Hanke, and C. Werner. Design and evaluation of novel blood incubation systems for in vitro hemocompatibility assessment of planar solid surfaces. J. Biomed. Mater. Res., Part B. 66: 379-390, 2003.

${ }^{33}$ Weisenberg, B. A., and D. L. Mooradian. Hemocompatibility of materials used in microelectromechanical systems: platelet adhesion and morphology in vitro. J. Biomed. Mater. Res. 60:283-291, 2002.

${ }^{34}$ Yoshioka, T., K. Tsuru, S. Hayakawa, and A. Osaka. Blood compatibility of stainless-steel and titanium immobilized with alginic acid layers. Mater. Res. Soc. Symp. Proc. 734:333-338, 2003.

${ }^{35}$ Zhang, F., E. T. Kang, K. G. Neoh, P. Wang, and K. L. Tan. Modification of $\mathrm{Si}(100)$ surface by the grafting of poly(ethylene glycol) for reduction in protein adsorption and platelet adhesion. J. Biomed. Mater. Res. 56:324-332, 2001. 